\title{
LA TERCERIZACIÓN Y EL RENDIMIENTO FINANCIERO: MUESTRA DE EMPRESAS ESTADOUNIDENSES, 2000-2009
}

\author{
Pedro L. Ortiz Santos ${ }^{1}$ \\ Ahmad H. Juma'h ${ }^{2}$
}

Recibido: 27/04/2014

Aprobado: 30/09/2015

\begin{abstract}
RESUMEN
Este estudio evaluó la relación entre tercerización y el desempeño financiero de las empresas en Estados Unidos de Norte América (2000-2009). Con este fin, se calculó el efecto de tercerización en dos variables: la rentabilidad y la liquidez. En el primer caso se utilizó el margen operacional antes de interés e impuestos, el retorno sobre activos y el retorno sobre patrimonio; mientras que en el segundo caso, se empleó la razón rápida, la razón de deuda a total de activos y la razón de deuda a patrimonio neto. Se utilizaron los cambios porcentuales en los parámetros financieros en el año de anuncio de tercerización $\left(t_{0}\right)$ y el año subsiguiente $\left(t_{1}\right)$, los cuales resultaron no significativos $(\alpha=0,05)$ para las empresas que tercerizan. También, no se encontró diferencias significativas $(\alpha=0,05)$ en la rentabilidad y liquidez de las empresas con anuncios de tercerización y aquellas sin ellos.

PALABRAS CLAVE: RENTABILIDAD, LIQUIDEZ, ANUNCIOS, CONTRATOS, CRISIS FINANCIERA, REDUCCIÓN DE COSTO, SEÑAL DE REORGANIZACIÓN.
\end{abstract}

\section{ABSTRACT}

This study evaluated the relation between outsourcing and firm's financial performance in the USA (2000-2009). To measure the effect of outsourcing on the company's profitability, operating profit margin before interest and taxes, return on assets, and return on equity were used. To measure the effect of outsourcing on the company's liquidity, quick ratio, total debt to total assets, and total debt to total equity were used. The study used the percentages of changes in financial parameters in the outsourcing year $\left(t_{0}\right)$ and subsequent year $\left(t_{1}\right)$, no indications of significance $(\alpha=0.05)$ were found. Also, no significant $(\alpha=0.05)$ results were found with respect to profitability and liquidity for companies with outsourcing announcements and those without announcements.

KEYWORDS: PROFITABILITY, LIQUIDITY, ANNOUNCEMENTS, CONTRACTS, FINANCIAL CRISIS, COST REDUCTION, REORGANIZATION SIGNAL.

1 Universidad del Turabo. Escuela de Negocios y Empresarismo, PO Box 3030, Gurabo, Puerto Rico, 00778; ortizp1@suagm.edu

2 Universidad Interamericana de Puerto Rico, Escuela de Economía, Apartado 191293, San Juan, Puerto Rico, 00919-1293; jumah@intermetro.edu 


\section{INTRODUCCIÓN}

Las empresas adoptan prácticas rutinarias (operativas frecuentes) $y$ otras a largo plazo (estratégicas) para maximizar su desempeño financiero. Entre dichas medidas se encuentra la tercerización, la cual está relacionada con los posibles ahorros en costos, la transferencia de riesgos, la obtención de recursos técnicos especializados, flexibilidad operacional y acceso a nuevas tecnologías (Andersson \& Pedersen, 2010; Minonne y Turner, 2012). Previo a la tercerización, la empresa determina qué funciones, actividades o servicios podrían ser adquiridos en el mercado. Además, se concentra en las actividades que fortalecen su ventaja competitiva, las cuales son difíciles de imitar por parte de los competidores, por lo que es esencial realizarlas internamente (Hamel \& Prahalad, 1990).

La tercerización comprende desde los componentes operacionales de la compañía (matriz, subsidiaria o inversión extrajera directa) hasta los acuerdos contractuales con otras empresas y en otras localidades. Existen dos variantes de la tercerización: el outsourcing (externalización) y el offshoring (deslocalización). El primero se refiere a la contratación (tercerización) en el mercado de una parte o la totalidad de una actividad o un servicio que la empresa adquiría internamente. Por su parte, el offshoring está asociado a las actividades que se trasladan a países con mano de obra más barata (Metters y Verma, 2008; Wernerheim, 2012; Bertrand y Mol, 2013).

El objetivo principal de tercerización es reducir costos (Lacity \& Hirsham 1993; Agrawal, Goswami, y Chatterjee 2010; Butler \& Callahan, 2012), lo cual es consistente con la teoría de costo de transacción (Coase, 1937; Williamson, 1975, 2008). Otros factores influyentes para establecer contratos de tercerización incluyen el fortalecimiento o desarrollo de una ventaja competitiva basada en recursos naturales y humanos (Wernerfelt, 1984; Barney 1991).

Por otra parte, la reducción de costos y la concentración en las actividades que fortalecen las ventajas competitivas de la empresa fundamentan la posible relación entre los anuncios de contratos de tercerización y el desempeño financiero, en las áreas de rentabilidad y liquidez; así como un vínculo con otros elementos tales como: la eficiencia operacional, el desarrollo de la competencia medular, la disponibilidad de recursos técnicos y especializados, la transferencia de riesgos, nuevas tecnologías y flexibilidad operacional (Kenney, Massini y Murtha, 2009; Doh, Bunyaratavej \& Hahn, 2009; Shan y Zhaohua, 2015).

La tercerización reduce la cantidad de actividades de la cadena de valor, con el objetivo de mantener niveles eficientes, así sea en las actividades realizadas en la empresa, en aquellas contratadas en el mercado o en ambas (Rottman y Lacity 2006). En este sentido, es pertinente señalar que el proceso es una reconfiguración de la empresa (Ikerionwu, Foley, Gray \& Edgar, 2014) y se apoya en las relaciones entre las partes establecidas en el acuerdo sobre la especificidad del activo, la incertidumbre y la frecuencia de las transacciones (Williamson, 1979, 1991).

La integración vertical de operaciones se sustenta en que la creación y protección de valor es más efectiva dentro de la empresa (Stuckey y White, 1993), si se compara a la efectividad alcanzada cuando los costos de producción son menores a los costos de adquirir el bien o servicio en el mercado (Coase, 1937). Si bien, la integración vertical continúa persiguiendo los mismos objetivos estratégicos, se diferencia en que todos los elementos para el desarrollo de una ventaja competitiva están dentro de la empresa.

El reducir las funciones y actividades de la cadena de valor, mediante tercerización, tiene como fin mantener las actividades medulares de la empresa. El manejo de la complejidad promueve varios modelos de relación en el contrato. En este escenario, la empresa adopta aquel en el que logra fomentar una alianza colaborativa entre la partes (Ebiringa y Kule, 2014).

El suministro de productos y servicios en las empresas ha evolucionado desde el abastecimiento de componentes básicos, a través de la integración vertical de la producción, el establecimiento de una subsidiaria o mediante 
la inversión extranjera directa, hasta la diversidad de las modalidades de tercerización (Wernerhem, 2012). Metters y Verma (2008) identificaron algunos factores que impulsan esta práctica: la efectividad de las tecnologías de información y comunicación, cambios en las regulaciones e incentivos para inversión en economías en desarrollo, así como la estandarización de procesos de manera global. Ejemplos de la migración de servicios internos o administrativos a otros países que cuentan con mano de obra más económica incluyen: recursos humanos, finanzas, contabilidad, procuraduría, cumplimientos y servicios al cliente (Contractor, Kumar, Kundu y Pedersen, 2010).

La perspectiva basada en recursos plantea que la empresa hace una composición de activos dirigida a desarrollar una ventaja competitiva sostenible (Barney, 1991; Grant, 1991). La adopción de esta estrategia tiene como objetivo afianzar la ventaja competitiva con una estructura operacional más eficiente. En este sentido, la transferencia de operaciones a otras localidades, cuya fuerza laboral es más económica, genera ahorros en mano de obra (Contractor et al., 2010). Metters (2008) identifica otros factores que incluyen conocimiento local, diferencias y sesgos culturales, riesgo del país, comunicación, temporadas, escalas, distancias y la moral de los empleados domésticos.

El supuesto de que las empresas buscan maximizar sus ganancias y su ventaja competitiva, las lleva a adoptar prácticas consistentes con sus competencias medulares. La tercerización es una práctica que se ha incrementado en las pasadas tres décadas, ante los cambios generados por la globalización en la producción de bienes y la prestación de servicios (Contractor et al., 2010; Hamel y Prahald, 1985).

Hope, Kang, Thomas y Vasvari, (2009), Juma'h, $(2004,2006)$ y Harrison y McMillan, (2006) han planteado que resulta difícil medir la tercerización con respecto al desempeño financiero utilizando los datos internos de las empresas que tercerizan. Los estudios empíricos congruentes con este tema presentan resultados mixtos y los trabajos anteriores son descriptivos, los cuales emplean estudios de caso o consideran los cambios de valor de acciones comunes de las empresas que tercerizan utilizando estudios de eventos. En algunos casos, se observan efectos positivos (Prezas, Simonyan y Vasudevan, 2010; Agrawal et al., 2010; Naru \& Truitt, 2013; Ikerionwu et al., 2014) y en otros, se indica que las empresas que tercerizan no han logrado mejorías en el desempeño financiero después de la tercerización (Gilley y Rasheed, 2000; Görg y Hanley, 2004; Manning, Levin y Schuerch, 2011 y Jensen y Pedersen, 2012). El presente artículo utiliza las cuentas de los estados financieros para las empresas que tercerizan para medir la relación entre la tercerización $y$ el rendimiento financiero.

Esta investigación evaluó la relación entre la tercerización y el rendimiento financiero (rentabilidad y liquidez) de las empresas estadounidenses. Para este análisis, se identificaron anuncios de contratos de tercerización de empresas estadounidenses, emitidos durante el periodo de 2000 a 2009. Además, con el propósito de medir el efecto de tercerización en la rentabilidad y en la liquidez de la empresa, se utilizaron el margen operacional antes de interés e impuestos, el retorno sobre activos y el retorno sobre patrimonio, en el primer caso; mientras que para la liquidez, se empleó la razón rápida, la razón de deuda a total de activos y la razón de deuda a patrimonio neto.

En este estudio, se consideran los cambios porcentuales en los parámetros en el año de anuncio de tercerización $\left(\mathrm{t}_{0}\right)$ y el año subsiguiente $\left(t_{1}\right)$. Según los resultados, no existen diferencias significativas entre las empresas con anuncios de tercerización y las empresas sin ellos en relación al rendimiento financiero.

\section{METODOLOGÍA}

El total de las empresas identificadas con anuncios de contratos de tercerización es de 245, las cuales han tenido 300 anuncios durante el periodo 1 de enero de 2000 a 31 de diciembre de 2009. Las fuentes de anuncios son las bases de datos "Business \& Company Resource Center (Infotrac)" e Interfaz Avanzado de Negocios: "Business Source Complete" (EBSCO), en las cuales se buscaron las siguientes palabras claves: "outsourcing", 
"offshoring", subcontracting 3 ". Utilizando los contenidos de los anuncios de tercerización, se identificaron los siguientes elementos: valor del contrato, duración del contrato, tercerización (outsourcing), beneficio u objetivo, nombre del suplidor y número de empleados trasladados o despedidos. Los anuncios de tercerización que fueron seleccionados como parte de la muestra tienen, por lo menos, tres de las variables antes indicadas. Para validar el contenido de los anuncios de tercerización, se verificó en la página web de cada empresa y con "Google Search" la existencia de información distinta al contenido de los anuncios. Los anuncios duplicados, sin fecha de inicio del contrato de tercerización, aquellos donde los contratos fueron cancelados durante el año establecido o el subsiguiente, los relacionados con servicios y noticias de proveedores fueron eliminados de la muestra.

En la comparación de la rentabilidad y la liquidez de las empresas que tercerizan antes y después de los anuncios de contratos de tercerización, se limitó al ámbito de las compañías públicas estadounidenses $y$ foráneas registradas para hacer negocios en el mercado de valores de Estados Unidos de América. No se consideraron instituciones financieras (bancos, aseguradoras) ni unidades gubernamentales; además, se excluyeron empresas cuya información financiera era incompleta. La selección final de la muestra utilizada para el análisis de los parámetros financieros consistió de 53 anuncios en 53 compañías.

Se estableció un grupo de control constituido por una empresa perteneciente a la misma industria para cada empresa seleccionada en la muestra, de similar tamaño de ventas y activos totales con el propósito de establecer una comparación. Las empresas del grupo de control fueron seleccionadas de la base de datos "Infotrac". Ninguna empresa fue incluida más de una vez para el grupo de control. Para asegurar que las compañías utilizadas en comparación no han tenido anuncios de tercerización en el periodo de estudio, se produjo una búsqueda en las bases de datos "Infotrac" y "EBSCO".

3 Términos en inglés, requeridos por el lenguaje de la base de datos

Ciencias Económicas 33-N²2: 2015 / 25-37 / ISSN: 0252-9521
Existen algunas posibilidades que limitan el presente estudio para la generalización de los resultados. Por ejemplo, las empresas en el grupo de control podrían tener contratos de tercerización y no anunciar dichos contratos, o bien, los dos grupos (que anuncian contratos de tercerización y de control) podrían tener otros eventos no anunciados que influyen en los rendimientos financieros en el mismo tiempo que el anuncio de contrato de tercerización.

Para medir el efecto de tercerización en la rentabilidad y en la liquidez de la empresa, en el primer caso, se utilizó el margen operacional antes de interés e impuestos, el retorno sobre activos y el retorno sobre patrimonio; mientras que para la liquidez, se empleó la razón rápida, la razón de deuda a total de activos y la razón de deuda a patrimonio neto. En este estudio se consideran los cambios porcentuales en los parámetros financieros en el año de anuncio de tercerización $\left(\mathrm{t}_{0}\right)$ y el año subsiguiente $\left(\mathrm{t}_{1}\right)$.

Los parámetros financieros utilizados en la medición del rendimiento de las empresas que tercerizan fueron extraídos de la base de datos "Standard \& Poor's Research Insight (Compustat)". El periodo de tiempo establecido para el análisis consiste en los tres años previos $\left(\mathrm{t}_{-3}, \mathrm{t}_{-2} \mathrm{y}\right.$ $t_{-1}$ ), el año de tercerización $\left(t_{0}\right)$ y un año después del anuncio $\left(t_{1}\right)$. Se señalaron los años $t_{0}$ y $t_{1}$ para medir el efecto del anuncio de tercerización a corto plazo. El análisis de los datos contables, mediante las razones financieras, se empleó con el propósito de comparar las métricas de desempeño. Así mismo, el análisis del coeficiente de correlación de Pearson fue utilizado con el objetivo de determinar la relación del valor del contrato con los resultados de todas las razones financieras. Para lograr normalidad con las variables a correlacionar, se ajustó el valor del contrato de tercerización mediante transformación logarítmica natural. Además, se utilizó la prueba $t$ apareada para comparar razones financieras entre periodos; $y$ la prueba t, para comparar los dos grupos (con anuncios de tercerización y sin estos). La selección de las pruebas estadísticas se fundamentó mediante las pruebas de normalidad Shapiro- Wilk y Sminorv-Kolmogorov. 


\section{RESULTADOS}

El gráfico 1 presenta la distribución de los 300 anuncios de contratos de tercerización utilizados para identificar las empresas que tercerizan durante el periodo (2000-2009).
El aumento en los contratos de tercerización entre el 2007 y 2009 es consistente con la crisis económica en muchos países, en particular en Estados Unidos de América.

\section{GRÁFICO 1 \\ ANUNCIOS DE TERCERIZACIÓN}

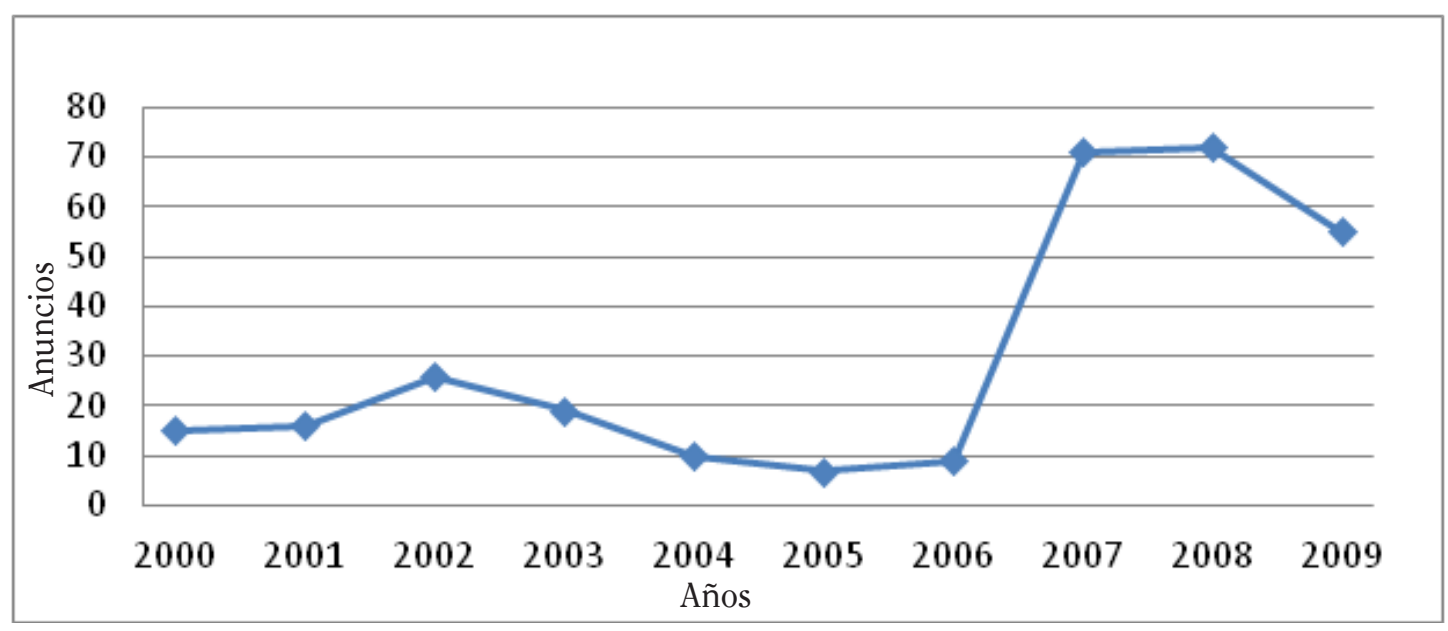

Fuente: elaboración propia basada en la recopilación de anuncios.

Las empresas con negocios en los Estados Unidos de América, Reino Unido, India, Alemania, Suecia, Australia y Suiza representan el
$79,6 \%$ de los anuncios de contratos de tercerización en la muestra (cuadro 1).

CUADRO 1

PAÍSES CON ANUNCIOS DE EMPRESAS QUE TERCERIZAN EN EL PERIODO 2000-2009

\begin{tabular}{lcc}
\hline País & Anuncios & $\%$ \\
\hline Estados Unidos de América & 141 & 47,0 \\
Reino Unido & 34 & 11,3 \\
India & 16 & 5,3 \\
Alemania & 16 & 5,3 \\
Suecia & 11 & 3,7 \\
Australia & 11 & 3,7 \\
Suiza & 10 & 3,3 \\
Total de anuncios que incluyen el país en el contenido & 239 & $79,6 \%$ \\
Total de anuncios & 300 & $100 \%$ \\
\hline
\end{tabular}

Fuente: elaboración propia basada en la recopilación de anuncios. 
Los sectores industriales con mayor representación en los anuncios fueron la manufactura (34\%, 103 anuncios), finanzas, seguros $y$ bienes raíces (28\%, 84 anuncios), transportación y utilidades $(15 \%, 45$ anuncios) y servicios (11,33\%, 34 anuncios). El remanente de los sectores fluctúan entre el 4,33\% y el 0,33\% (34 anuncios). Además, en el sector de manufactura, los tipos de negocios predominantes lo constituyen las farmacéuticas y los fabricantes de autos, aviones, computadoras y componentes electrónicos. Por su parte, los bancos y las aseguradoras dominan el sector de finanzas. Finalmente, las líneas aéreas y los agentes de viajes sobresalen en transporte; mientras que los planes médicos y servicios de salud, en el sector de servicios.

La actividad contratada predominante en los anuncios fueron las relacionadas con tecnologías de información $(78 \%$ de los anuncios), manufactura, procesos internos o administrativos contratados (business process outsourcing, BPO, por sus siglas en inglés) y distribución y logística.

Las actividades relacionadas con tecnologías de información comprenden el mantenimiento de hardware, mantenimiento de software, administración o desarrollo de infraestructuras, instalación de sistemas, desarrollo de nuevos programas, servicios de redes, seguridad y resguardo de emergencias, análisis de aplicaciones, transmisión de datos y voz, operación de sistemas, apoyo de usuarios finales y soluciones "e-business". Además, el proceso interno o administrativo contratado (BPO) incluyó actividades en los departamentos de recursos humanos, finanzas, contabilidad, actividades de facturación y cobros, procesamiento y trámite de financiamiento hipotecario, procesamiento de cheques o imágenes de cheques y preparación de alimentos.

Los proveedores con mayores contratos en los 300 anuncios son "International Business Machines" (IBM), con 65 contratos; "Electronic Data Systems Corp"(EDS), con 27 contratos y "Computer Sciences Corp"(CSC), con 24 contratos. Con los 116 anuncios indicados para IBM, EDS y CSC, las empresas influyeron en 29,963 puestos de trabajos entre transferidos o despedidos. Así mismo, la media del valor de los contratos fue mayor para EDS (\$1,258 millones) que para los otros suplidores. Un elemento en común entre los tres proveedores es la duración del contrato, siendo la media de 7 años. Esto indica que la relación entre las dos partes es a largo plazo.

Según el contenido de los 300 anuncios que especificaban reducción o transferencias de empleados, los diez proveedores con mayor número de anuncios generaron 33,309 transferencias o despedidos (ver gráfico 2). Esto es equivalente al $69 \%$ de los puestos de trabajos afectados. Los restantes 88 proveedores repercutieron en el remanente de 15,148 puestos de trabajo(31\%). Además, los tres proveedores IBM, EDS y CSC ofrecen servicios a las empresas multinacionales más grandes en términos de ventas.

El valor promedio de los contratos asciende a $\$ 755$ millones. El promedio general de duración es de 6,4 años para los contratos identificados como iniciales y 6,1 años en contratos de renovación.

GRÁFICO 2

CONTRATISTAS CON MAYOR NÚMERO DE CONTRATOS

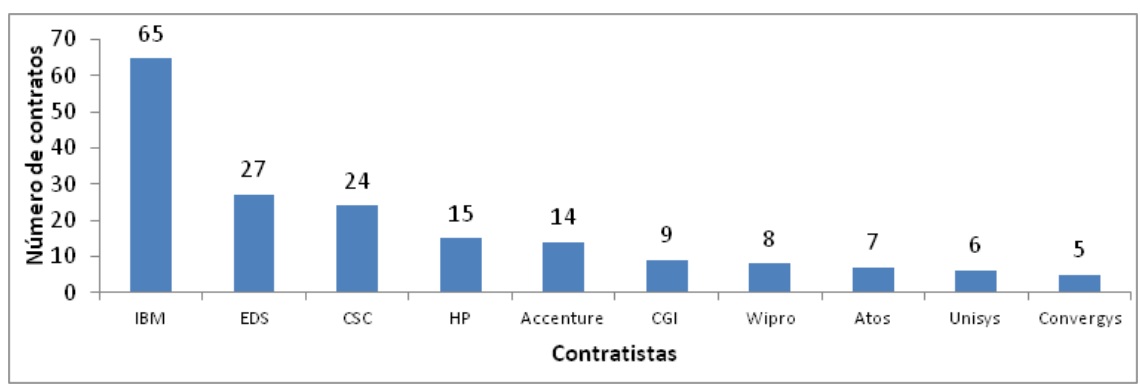

Fuente: elaboración propia basada en la recopilación de anuncios. 
La reducción de costos fue la principal expectativa de beneficio en 109 anuncios, la cual influyó en el control de los costos directos y el manejo de los gastos de capital. Según lo indicado en los anuncios, la efectividad en este beneficio está centrada en la utilización de la tecnología de información y en los procesos de negocios contratados; mientras que los otros beneficios esperados incluían mejoras y actualización de sistemas, eficiencia, concentración en competencia medular e integración y estandarización de sistema. En el cuadro 2, se presentan 163 anuncios de los 195 con beneficios esperados.

CUADRO 2

OBJETIVOS, EMPLEADOS AFECTADOS Y ACTIVIDADES CONTRATADAS

\begin{tabular}{lcrrrl}
\hline Objetivos indicados en los anuncios & $\begin{array}{r}\text { Frecuencia } \\
\text { (Anuncios) }\end{array}$ & $\%$ & $\begin{array}{r}\text { Empleos } \\
\text { Afectados }\end{array}$ & $\%$ & Actividad Contratadas \\
\hline Reducción de costos & 109 & 66,5 & 22,385 & 66,0 & TI (80), BPO (24), Mfra (4), Log (1) \\
Mejoras y actualización de Sistemas & 20 & 12,5 & 3,187 & 9,4 & TI (14), BPO (6) \\
Eficiencia & 13 & 8,0 & 1,020 & 3,0 & TI (13) \\
Concentrar en competencia medular & 11 & 6,8 & 7,055 & 20,8 & TI (8), BPO (2), Dist (1) \\
Integración y estandarización de sistemas & 10 & 6,2 & 260 & 0,8 & TI (9), BPO (1) \\
\cline { 2 - 5 } & 163 & 100 & 33907 & 100 & \\
\hline
\end{tabular}

Leyenda: BPO: Procesos de Negocio Contratado; TI: Tecnología de Información, Mfra. Manufactura; Log: Logística; Dist: Distribución.

Fuente: elaboración propia basada en la recopilación de anuncios.

El margen operacional promedio antes de interés e impuestos tiene una reducción de $9,28 \%$ y de $5,16 \%$ en los periodos $t_{-3}$ a $_{0} y t_{-1}$ a $\mathrm{t}_{0}$, respectivamente. No obstante, en el periodo posterior, se observa un leve aumento de 0,54 $\%$ (cuadro 3). Este incremento puede estar relacionado con la reducción del gasto de nómina y la reducción de gastos operacionales de las actividades transferidas. La correlación Pearson entre el logaritmo natural del valor de contrato de tercerización y margen operacional antes de intereses e impuestos reflejó coeficiente de correlación Pearson de -0,3 $(\alpha=0,09)$.

\section{CUADRO 3
MARGEN OPERACIONAL ANTES DE IMPUESTOS E INTERESES (EN PORCENTAJES)}

\begin{tabular}{lccccccccc}
\hline & \multicolumn{4}{c}{ Empresas con anuncios de tercerización } & \multicolumn{3}{c}{ Grupo de comparación } \\
\hline Periodo & $\mathrm{N}$ & Mínimo & Máximo & Media & $\begin{array}{c}\text { Desviación } \\
\text { Estándar }\end{array}$ & Mínimo & Máximo & Media & $\begin{array}{c}\text { Desviación } \\
\text { Estándar }\end{array}$ \\
\hline $\mathrm{t}_{-3}$ & 53 & 2,299 & 44,241 & 15,733 & 9,268 & $-25,787$ & 43,697 & 14,735 & 12,345 \\
$\mathrm{t}_{-2}$ & 53 & $-2,043$ & 41,827 & 15,323 & 10,006 & $-15,799$ & 44,355 & 14,363 & 11,239 \\
$\mathrm{t}_{-1}$ & 53 & $-2,646$ & 41,827 & 15,050 & 10,171 & $-14,911$ & 45,264 & 14,284 & 11,962 \\
$\mathrm{t}_{0}$ & 53 & $-0,959$ & 39,702 & 14,273 & 10,306 & $-14,616$ & 38,842 & 13,842 & 12,166 \\
$\mathrm{t}_{1}$ & 53 & $-13,940$ & 39,730 & 14,350 & 11,353 & $-19,677$ & 36,793 & 14,150 & 11,785 \\
\hline
\end{tabular}

"t $t_{-3}$ ", "t $t_{-2}$ " $y$ " $t_{-1}$ "son los tres años antes del anuncio de tercerización, " $t_{0}$ " es el año de tercerización y " $t_{1}$ " es el año siguiente al anuncio de tercerización.

Fuente: elaboración propia basada en los datos de "Compustat". 
Para el grupo de comparación, el margen operacional antes de impuestos e intereses fue porcentualmente menor en el año de tercerización que en los años anteriores. En el siguiente año este mismo parámetro muestra un aumento. Los cambios porcentuales del margen operacional antes de impuestos e intereses para el periodo $t_{1}$ de las empresas que anuncian la tercerización y aquellas que no son de 3,20\%, $y$ de 2,23\%, respectivamente. Este es un comportamiento similar a las empresas con anuncios de tercerización.

El cambio en el retorno sobre activos entre los años anteriores, el año de tercerización $y$ el año siguiente mide la generación de ganancias y la administración efectiva de la inversión. Las empresas con anuncios de tercerización y el grupo de comparación presentan una reducción en el promedio de retorno sobre activos en los tres años anteriores a la tercerización (cuadro 4). Las empresas del grupo de control presentan una breve mejoría en el indicador; sin embargo, en el año $t_{1}$, el indicador es menor que en el año $\mathrm{t}_{0}$. La relación entre logaritmo natural del valor de contrato de tercerización y retorno sobre activos tiene coeficiente de correlación Pearson de $-0,35(\alpha=0,048)$.

CUADRO 4

RETORNO SOBRE ACTIVOS (EN PORCENTAJES)

\begin{tabular}{lcrrrrrrrr}
\hline & \multicolumn{4}{c}{ Empresas con anuncios de tercerización } & \multicolumn{3}{c}{ Grupo de comparación } \\
\hline Periodo & $\mathrm{N}$ & Mínimo & Máximo & Media & $\begin{array}{c}\text { Desviación } \\
\text { Estándar }\end{array}$ & Mínimo & Máximo & Media & $\begin{array}{c}\text { Desviación } \\
\text { Estándar }\end{array}$ \\
\hline $\mathrm{t}_{-3}$ & 53 & $-5,889$ & 21,090 & 7,710 & 5,588 & $-12,071$ & 24,544 & 7,391 & 6,735 \\
$\mathrm{t}_{-2}$ & 53 & $-11,788$ & 21,563 & 6,885 & 6,392 & $-12,833$ & 21,090 & 6,766 & 5,937 \\
$\mathrm{t}_{-1}$ & 53 & $-7,977$ & 21,090 & 6,837 & 6,377 & $-41,393$ & 16,818 & 5,266 & 8,823 \\
$\mathrm{t}_{0}$ & 53 & $-12,270$ & 22,265 & 5,841 & 6,940 & $-22,501$ & 34,083 & 5,638 & 8,041 \\
$\mathrm{t}_{1}$ & 53 & $-31,591$ & 24,289 & 5,626 & 9,285 & $-25,819$ & 24,088 & 4,485 & 8,106 \\
\hline
\end{tabular}

" $t_{-3}$ ", " $t_{-2}$ " y " $t_{-1}$ "son los tres años antes del anuncio de tercerización, " $t_{0}$ " es el año de tercerización y " $t_{1}$ " es el año siguiente al anuncio de tercerización.

Fuente: elaboración propia basada en los datos de "Compustat".

Existe una tendencia a la reducción del retorno sobre patrimonio para las empresas, tanto las que realizaron anuncios, como las del grupo de control (cuadro 5). La relación entre logaritmo natural del valor de contrato de tercerización y retorno sobre patrimonio tiene coeficiente de correlación Pearson de $-0,13,(\alpha=0,48)$. 
CUADRO 5

RETORNO SOBRE PATRIMONIO (EN PORCENTAJES)

\begin{tabular}{lcrrrrrrrr}
\hline & \multicolumn{4}{c}{ Empresas con anuncios de tercerización } & \multicolumn{3}{c}{ Grupo de comparación } \\
\hline Periodo & $\mathrm{N}$ & Mínimo & Máximo & Media & $\begin{array}{c}\text { Desviación } \\
\text { Estándar }\end{array}$ & Mínimo & Máximo & Media & $\begin{array}{c}\text { Desviación } \\
\text { Estándar }\end{array}$ \\
\hline $\mathrm{t}_{-3}$ & 53 & $-11,879$ & 93,827 & 20,597 & 17,514 & $-94,745$ & 80,414 & 17,412 & 22,002 \\
$\mathrm{t}_{-2}$ & 53 & $-28,756$ & 61,942 & 17,540 & 15,220 & $-342,530$ & 81,230 & 12,437 & 52,205 \\
$\mathrm{t}_{-1}$ & 53 & $-22,111$ & 59,489 & 17,774 & 16,643 & $-36,752$ & 132,263 & 16,297 & 21,879 \\
$\mathrm{t}_{0}$ & 53 & $-82,057$ & 58,083 & 12,554 & 25,290 & $-113,274$ & 344,693 & 19,646 & 54,996 \\
$\mathrm{t}_{1}$ & 53 & $-72,639$ & 91,600 & 15,407 & 27,333 & $-70,229$ & 150,810 & 13,170 & 31,485 \\
\hline
\end{tabular}

"t $\mathrm{t}_{-3}$ ", " $\mathrm{t}_{-2}$ " $\mathrm{y}$ " $\mathrm{t}_{-1}$ "son los tres años antes del anuncio de tercerización, " $\mathrm{t}_{0}$ " es el año de tercerización y " $\mathrm{t}_{1}$ " es el año siguiente al anuncio de tercerización.

Fuente: elaboración propia basada en los datos de "Compustat".

Las pruebas $t$ indican que, entre las empresas que han tenido anuncios de tercerización y aquellas sin ellos, no existen diferencias $(\alpha=0,05)$ en cuanto a los indicadores retorno sobre activos y retorno sobre patrimonio.

Para las empresas que tercerizaron, la liquidez examinada a través de la razón rápida, presenta una tendencia decreciente entre los periodos $t_{-3}$ a $t_{-1}$. Para los periodos $t_{0} y t_{1}$, el cambio porcentual para la razón rápida fue de $5,48 \%$ y de 5,1 \% respectivamente. El grupo de comparación reflejó una reducción entre los periodos $\mathrm{t}_{-3}$ a $\mathrm{t}_{1}$ (cuadro 6).

\section{CUADRO 6 \\ RAZÓN RÁPIDA}

\begin{tabular}{lccccccccc}
\hline & \multicolumn{4}{c}{ Empresas con anuncios de tercerización } & \multicolumn{3}{c}{ Grupo de comparación } \\
\hline Periodo & $\mathrm{N}$ & Mínimo & Máximo & Media & $\begin{array}{c}\text { Desviación } \\
\text { Estándar }\end{array}$ & Mínimo & Máximo & Media & $\begin{array}{c}\text { Desviación } \\
\text { Estándar }\end{array}$ \\
\hline $\mathrm{t}_{-3}$ & 53 & 0,327 & 4,441 & 1,101 & 0,754 & 0,210 & 6,568 & 1,161 & 0,981 \\
$\mathrm{t}_{-2}$ & 53 & 0,319 & 4,441 & 1,083 & 0,727 & 0,260 & 4,457 & 1,070 & 0,658 \\
$\mathrm{t}_{-1}$ & 53 & 0,153 & 2,662 & 0,985 & 0,516 & 0,260 & 4,606 & 1,035 & 0,680 \\
$\mathrm{t}_{0}$ & 53 & 0.122 & 2,798 & 1,039 & 0,527 & 0,210 & 2,648 & 1,055 & 0,542 \\
$\mathrm{t}_{1}$ & 53 & 0,302 & 3,110 & 1,092 & 0,513 & 0,207 & 2,317 & 1,019 & 0,469 \\
\hline
\end{tabular}

" $t_{-3}$ ", " $t_{-2}$ " $y$ " $t_{-1}$ "son los tres años antes del anuncio de tercerización, " $t_{0}$ " es el año de tercerización y " $t_{1}$ " es el año siguiente al anuncio de tercerización.

Fuente: elaboración propia basada en los datos de "Compustat".

Durante el periodo $t_{0}$ a $t_{1}$, la razón entre deuda $y$ total de activos no demuestra cambios notables para las empresas que tercerizaron, $y$ para el grupo de control (cuadro 7). La relación entre logaritmo natural del valor de contrato de tercerización y razón rápida tiene coeficiente de correlación Pearson de -0,28 $(\alpha=0,12)$. Mientras que, cuando se mide la relación entre el primer parámetro y razón entre deuda y total de activos, existe un coeficiente de correlación Pearson de 0,15 $(\alpha=0,39)$. 


\section{CUADRO 7 \\ RAZÓN DE DEUDA A TOTAL DE ACTIVOS (EN PORCENTAJES)}

\begin{tabular}{lccccccrrrr}
\hline & \multicolumn{4}{c}{ Empresas con anuncios de tercerización } & \multicolumn{3}{c}{ Grupo de comparación } \\
\hline Periodo & $\mathrm{N}$ & Mínimo & Máximo & Media & $\begin{array}{c}\text { Desviación } \\
\text { Estándar }\end{array}$ & Mínimo & Máximo & Media & $\begin{array}{c}\text { Desviación } \\
\text { Estándar }\end{array}$ \\
\hline $\mathrm{t}_{-3}$ & 53 & 1,621 & 67,354 & 25,737 & 13,993 & 0,309 & 64,158 & 21,904 & 15,279 \\
$\mathrm{t}_{-2}$ & 53 & 3,813 & 65,727 & 26,413 & 14,447 & 0,000 & 76,613 & 22,691 & 15,098 \\
$\mathrm{t}_{-1}$ & 53 & 3,920 & 63,562 & 27,395 & 14,548 & 0,000 & 112,278 & 23,913 & 17,954 \\
$\mathrm{t}_{0}$ & 53 & 2,355 & 66,695 & 26,952 & 15,108 & 0,000 & 79,814 & 23,288 & 16,191 \\
$\mathrm{t}_{1}$ & 53 & 1,777 & 64,768 & 26,992 & 14,802 & 0,000 & 79,684 & 25,135 & 15,770 \\
\hline
\end{tabular}

" $t_{-3}$ ", " $t_{-2}$ " y " $t_{-1}$ "son los tres años antes del anuncio de tercerización, " $t_{0}$ " es el año de tercerización y " $t_{1}$ " es el año siguiente al anuncio de tercerización.

Fuente: elaboración propia basada en los datos de "Compustat".

Para todas las empresas en la muestra, la razón de deuda sobre patrimonio neto indica estabilidad en la deuda de largo plazo entre los periodos $\mathrm{t}_{-3}$ a $\mathrm{t}_{-1}$ (cuadro 8 ). Además, la relación entre logaritmo del valor de contrato de tercerización y razón entre deuda y patrimonio neto tiene coeficiente de correlación Pearson de $-0,11(\alpha=0,56)$.

CUADRO 8

RAZÓN DE DEUDA SOBRE PATRIMONIO NETO (EN PORCENTAJES)

\begin{tabular}{lrrrrrrrrr}
\hline & \multicolumn{4}{c}{ Empresas con anuncios de tercerización } & \multicolumn{3}{c}{ Grupo de comparación } \\
\hline Periodo & $\mathrm{N}$ & Mínimo & Máximo & Media & $\begin{array}{c}\text { Desviación } \\
\text { Estándar }\end{array}$ & Mínimo & Máximo & Media & $\begin{array}{r}\text { Desviación } \\
\text { Estándar }\end{array}$ \\
\hline $\mathrm{t}_{-3}$ & 53 & 0,000 & 429,802 & 76,314 & 82,186 & 0,000 & 494,112 & 63,900 & 93,031 \\
$\mathrm{t}_{-2}$ & 53 & 0,000 & 469,335 & 85,762 & 91,499 & $-2327,807$ & 304,994 & 13,371 & 334,021 \\
$\mathrm{t}_{-1}$ & 53 & 0,000 & 531,000 & 91,358 & 105,863 & $-240,916$ & 330,629 & 55,702 & 82,900 \\
$\mathrm{t}_{0}$ & 53 & 0,000 & 1559,594 & 114,548 & 230,060 & $-145,520$ & 873,925 & 82,325 & 155,459 \\
$\mathrm{t}_{1}$ & 53 & 0,000 & 2250,555 & 132,310 & 317,597 & $-204,160$ & 1970,531 & 126,303 & 341,722 \\
\hline
\end{tabular}

"t $t_{-3}$ ", " $t_{-2}$ " y " $t_{-1}$ "son los tres años antes del anuncio de tercerización, " $t_{0}$ " es el año de tercerización y " $t_{1}$ " es el año siguiente al anuncio de tercerización.

Fuente: elaboración propia basada en los datos de "Compustat".

La liquidez se evaluó mediante la aplicación de pruebas paramétricas a las razones financieras de razón rápida, razón de deuda a patrimonio neto y razón de deuda a activos totales. Los cambios porcentuales de la muestra indican un resultado positivo para el periodo $t_{1}$ en todas las variables de liquidez. Los cambios para razón rápida, razón de deuda a patrimonio y razón de deuda a total de activos son $5,1 \%, 15,56 \%$ y $1,5 \%$, respectivamente. Un cambio favorable en la liquidez (razón rápida) se observa en las empresas que tercerizan, al compararlas con el grupo de control en el periodo $t_{1}$. El cambio porcentual positivo fue de $0,1 \%$ para las empresas que contratan, mientras que hubo un cambio porcentual de $-3,41 \%$ para el grupo de comparación.

Las pruebas $t$ indican que no existe diferencias en cuanto a liquidez por efectos de la duración y valor del contrato entre las empresas que han tenido anuncios de 
tercerización y aquellas que no. Los valores $\mathrm{p}$ evidencian que no hay diferencias entre las empresas con anuncios de tercerización y sin ellos. El estadístico eta cuadrado indica que las diferencias entre las medias alrededor del evento resultan pequeñas (eta cuadrado: 0.014, 0.024, 0.000, para razón rápida, razón deuda a patrimonio neto, $y$ razón deuda a total activos, respectivamente).

\section{CONCLUSIONES}

La tercerización es un proceso para reducir costos operacionales y mejorar el desempeño financiero de la empresa. El objetivo de este artículo ha sido verificar el efecto de la tercerización en la rentabilidad y la liquidez de las empresas estadounidenses que han tenido al menos un anuncio de tercerización en el periodo (2000-2009).

Los parámetros financieros señalaban tendencias decrecientes entre los años anteriores al año de los anuncios de tercerización. Al periodo siguiente del anuncio, los índices de margen operacional antes de interés e impuestos y retorno sobre patrimonio reflejaron un pequeño incremento. Esto es consistente con el argumento de que la tercerización es parte de una estrategia o reestructuración que busca la mejoría en la cadena de valor. El efecto a corto plazo, en particular el segundo año $t_{1}$, podría indicar mejor utilización de los recursos. No se observa mejora en los índices en el año $t_{0}$, lo cual posiblemente se deba al efecto de los costos de transacción en que incurren las empresas por el cambio en la cadena de valor. Esto es consistente con los trabajos de Lacity \& Hirsham (1993), Agrawal, Goswami, y Chatterjee (2010) y Butler y Callahan (2012).

La razón rápida muestra una tendencia decreciente en los años anteriores al año del anuncio, logrando un leve incremento en el año $t_{0} y$ en el año $t_{1}$. Por otra parte, la razón entre la deuda y total de activos se mantuvo estable durante los periodos evaluados. Finalmente, la relación entre la deuda y patrimonio neto presentó un incremento. En resumen, la liquidez podría indicar que la tercerización se relaciona con el manejo de los activos a corto plazo; esto es consistente con la perspectiva basada en recursos (Barney, 1991; Grant, 1991; Narayanan, Narasimhan y Schoenherr, 2015).

Los resultados indican que no existen diferencias significativas $(\alpha=0,05)$ entre las empresas con anuncios de tercerización y aquellas sin ellos. Esto podría indicar que la tercerización es una alternativa para mejorar la eficiencia de la compañía, pero no es la única práctica. Una posible explicación se relaciona con que las empresas, cuando enfrentan dificultades financieras, utilizan los recursos internos o del mercado, independientemente de los anuncios hechos. La tercerización podría estar relacionada con la reorganización de las empresas, en particular después de enfrentar una crisis financiera (Barrar y Gervais, 2006).

El vínculo entre la tercerización y el rendimiento financiero de la empresa todavía es un tópico fértil y requiere más estudios que comparen las empresas que emplean esta práctica $y$ aquellas que no la utilizan. Para futuras investigaciones, se recomienda (1) el uso de una muestra más amplia y que incorpore a empresas de otros países, (2) la comparación entre industrias, (3) incorporar mayor cantidad de variables financieras y (4) comparar entre tercerización que utiliza los servicios de proveedores nacionales contra proveedores internacionales.

\section{REFERENCIAS}

Agrawal, S., Goswami, K., y Chatterjee, B. (2010). The evolution of offshore outsourcing in India. Global Business Review, 11, 239256. doi: 10.1177/097215091001100208.

Andersson , U., y Pedersen, T. (2010). Organizational design mechanisms for the R\&D function in a world of offshoring. Scandinavian Journal of Management, $26(4), 431-438$. doi: $10.1016 / \mathrm{j}$. scaman.2010.09.006.

Barney, J. (1991). Firm Resources and Sustained Competitive Advantage. Journal of Management, 17(1): 99-12.

Barrar, P. y Gervais, R. (2006). Global Outsourcing Strategies: an International 
Reference on Effective Outsourcing Relationships. Gower Publishing.

Bertrand, O., y Mol, M. J. (2013). The antecedents and innovation effects of domestic and offshore R\&D outsourcing: The contingent impact of cognitive distance and absorptive capacity. Strategic Management Journal, 34(6), 751-760. Doi:10.1002/smj.2034.

Butler, M. y Callahan, C. M. (2012). Human resource outsourcing: Market and operating performance effects of administrative HR functions. Journal of Business Research, 67(2), 218-224. doi. org/10.1016/j.jbusres.2012.09.026.

Coase, R. H. (1937). The nature of the firm. Economica, 4(16), 386-405.

Contractor, F. J., Kumar,V., Kundu, S.K., \& Pedersen, T. (2010). Reconceptualizing the Firm in a World of Outsourcing and Offshoring: The Organizational and Geographical Relocation of HighValue Company Functions. Journal of Management Studies, 47(8), 1417-1433. doi: 10.1111/j.1467-6486.2010.00945.x.

Doh, J., Bunyaratavej, K., \& Hahn, E.D. (2009). Separable but not equal: The location determinants of discrete services offshoring activities. Journal of International Business Studies, 40(6): 936-943. doi: 10.1057/jibs.2008.89.

Ebiringa, T. O. \& kule, L. (2014). Offshoring for sustainable value management. Serbian Journal of Management, 9(1), 105-119. doi: 10.5937/sjm9-5626.

Gilley, K., \& Rasheed, A. (2000). Making more by doing less: An analysis of outsourcing and its effects on firm performance. Journal of Management, 26: 763-790. doi: 10.1016/ S0149-2063(00)00055-6.

Görg, H., \& Hanley, A. (2004). Does outsourcing increase profitability? Economic and Social Review, 35, 267-287.

Grant, R. M. (1991). The Resource-Based Theory of Competitive Advantage: Implications for Strategy Formulation. California Management Review, 33(3), 144-135. doi: $10.2307 / 41166664$.

Hamel, G., \& Prahalad, C. K. (1990), "Core
Competence of the Corporation", Harvard Business Review, May-June, pp. 79-91.

Hamel, G., y Prahalad, C.K. (1985). Do you really have a global strategy? Harvard Business Review, 63(4), 139-148.

Harrison, A. E., y McMillan, M.S. (2006). Dispelling Some Myths About Offshoring. The Academy of Management Perspective, 20(4), 6-22.

Hope, O-K., Kang, T., Thomas, W.B. y Vasvari, F. (2009). The effects of SFAS 131 geographic segment disclosure by US multinational companies on the valuation of foreign earning. Journal of International Business Studies, 40(3), 421-443.

Ikerionwu, C., Foley, R., Gray, E., y Edgar, D. (2014). Business Process Outsourcing Service Providers' Perception of Performance and Performance Measurement. Journal of Economics \& Engineering, 5(2), 5-10. Doi:10.7813/ jee.2014/5-2/1.

Jensen, P., y Pedersen, T. (2012). Offshoring and international competitiveness: Antecedents of offshoring advanced tasks. Journal of the Academy of Marketing Science, 40(2), 313-328. doi: 10.1007/ s11747-011-0286-x.

Juma'h, A. H. (2004). The investor's perceptions of outsourcing announcements. Économies et Sociétés, Économie et gestion des services, 6(10), 1807-1829.

Juma'h, A. H. (2006). The Materiality of Outsourcing, En P. Barra y R. Gervies (Ed.). Global Outsourcing Strategies: An International Reference on Effective Outsourcing Relationship (195-208). Gower Publishing.

Kenney, M., Massini, S., y Murtha, T. P. (2009). Offshoring administrative and technical work: New fields for understanding the global enterprise. Journal of International Business Studies, 40(6), 887-900. doi: 10.1057/jibs.2009.22.

Lacity, M. C., y Hirschheim, R. A. (1993). Information Systems Outsourcing; Myths, Metaphors, and Realities. John Wiley \& Sons, Inc. Doi: 10.1002/sres.3850120117.

Manning, S., Lewin, A. Y., y Schuerch, M. (2011). 
The stability of offshore outsourcing relationships. Management International Review, 51(3): 381-406. doi: 10.1007/ s11575-011-0081-4.

Metters, R. (2008). A typology of offshoring and outsourcing in electronically transmitted services. Journal of Operations Management, 26(2), 198-211. doi: 10.1016/j.jom.2007.02.004.

Metters, R., y Verna R. (2008), History of offshoring knowledge services. Journal of Operations Management, 26(2), 141-147. doi: 10.1016/j.jom.2007.02.012.

Minonne, C. y Turner, G. (2012). Business Process Management-Are You Ready for the Future?. Knowledge and Process Management, 19(3), 111-120. doi: 10.1002/kpm.1388.

Narayanan, S., Narasimhan, R., y Schoenherr, T. (2015). Assessing the contingent effects of collaboration on agility performance in buyer-supplier relationships. Journal of Operations Management, 33, 140-154. doi: 10.1016/j.jom.2014.11.004.

Naru, H. y Truitt, T. (2013). Can Partial Offshoring Contribute Growth of Multinational Electronic Manufacturing Service Comapnies in the United States?. International Journal of Applied Management and Technology, 12(1), 16-29. DOI:10.5590/IJAMT.2013.12.1.02.

Prezas, A., Simonyan, K., y Vasudevan, G. (2010). Does Offshoring create value for shareholders? Review of Financial Economics, 19(4), 179-191. Doi: 10.1016/j. rfe.2010.05.001.

Rottman, J. W., y Lacity, M. C. (2006). Proven Practices for Effectively Offshoring IT
Work. MIT Sloan Management Review, 47(3), 56-63.

Shan, L., y Zhaohua, D. (2015),"Understanding knowledge management capability in business process outsourcing", Management Decision, 53 (1), 124 - 138. doi: 10.1108/MD-04-2014-0197.

Stuckey, J., \& White, D. (1993). When and when not to vertically integrate. Sloan Management Review, Spring ,71-83.

Wernerfelt, B. (1984). A Resource-based View of the Firm. Strategic Management Journal, 5, 171-180. doi: 10.1002/smj.4250050207.

Wernerheim, C. M. (2012). Productivity Performance and the International Outsourcing of Services by Canadian Industry. International Trade Journal, 26(5), 413-435. doi: 10.1080/08853908.2012.721089.

Williamson, O. E. (1975). Markets and hierarchies: Analysis and antitrust implications. New York.

Williamson, O. E. (1979). TransactionCost Economics: The Governance of Contractual Relations. Journal of Law and Economics, 22(2), 23-261.

Williamson, O. E. (1991). Comparative economic organization: The analysis of discrete structural alternatives. Administrative science quarterly, 36(2), 269-296. doi: $10.2307 / 2393356$.

Williamson, O. E. (2008). Outsourcing: Transaction Cost Economics and Supply Chain Management. Journal of Supply Chain Management: A Global Review of Purchasing \& Supply, 44(2), 5-16. doi: 10.1111/j.1745-493X.2008.00051.x. 
